\title{
Expression of two human acyl-CoA:diacylglycerol acyltransferase isozymes in yeast and selectivity of microbial inhibitors toward the isozymes
}

\author{
Junji Inokoshi ${ }^{1}$, Kyosuke Kawamoto ${ }^{1}$, Yoichi Takagi ${ }^{1}$, Maki Matsuhama ${ }^{1}$, Satoshi Ōmura ${ }^{2}$ \\ and Hiroshi Tomoda ${ }^{1}$ \\ Two isozymes for human acyl-coenzyme A:diacylglycerol acyltransferase (DGAT), DGAT1 and DGAT2, were independently \\ expressed in DGAT-deficient Saccharomyces cerevisiae to establish DGAT1- and DGAT2-S. cerevisiae. The selectivity of DGAT \\ inhibitors of natural origin towards the isozymes was assessed in enzyme assays using the microsomal fractions prepared from \\ DGAT1- and DGAT2-S. cerevisiae. Amidepsines and xanthohumol inhibited DGAT1 and DGAT2 with similar potency, whereas \\ roselipins were found to inhibit DGAT2 selectively.
}

The Journal of Antibiotics (2009) 62, 51-54; doi:10.1038/ja.2008.5; published online 9 January 2009

Keywords: acyl-CoA:diacylglycerol acyltransferase; amidepsine; DGAT; isozyme; roselipin; triacylglycerol; xanthohumol

\section{INTRODUCTION}

Triacylglycerol (TG) is the major energy-storage form of long-chain fatty acids in animals. ${ }^{1,2}$ TG synthesis is important in many biological processes, including intestinal fat absorption, fat storage in adipocytes and energy metabolism in muscle, but excessive accumulation of TG in adipocytes as a result of a fat-rich diet or sedentary lifestyle causes obesity.

Acyl-coenzyme A (CoA):diacylglycerol acyltransferase (DGAT, EC2.3.1.20) is a membrane-bound enzyme that catalyzes TG formation by acyl esterifications of diacylglycerol. Two biological pathways for TG synthesis, the glycerol phosphate pathway and the monoacylglycerol pathway, have been reported. These pathways form diacylglycerol (DG), which in turn is acylated by DGAT to form TG. ${ }^{3}$ Recent molecular biological studies have revealed the existence of two different DGAT isozymes, DGAT1 and DGAT2, ${ }^{4-6}$ in mammals, and extensive studies including biological experiments and knockout mice have shown that these isozymes have different functions in mammals. ${ }^{7-13}$ Increased DGAT2 activity in the liver causes hepatic steatosis, whereas DGAT1 plays a role in very low-density lipoprotein (VLDL) synthesis in the liver and increases plasma VLDL concentration. Furthermore, newborn DGAT2-deficient mice die within hours of birth, whereas DGAT1-deficient mice are viable and have a modest reduction in tissue TG. Therefore, it is important to determine the selectivity of inhibitors towards the two DGAT isozymes for developing them as pharmaceutical drugs.

Our research group conducted an enzyme assay involving rat liver microsomes to discover several DGAT inhibitors from natural sources, including fungal amidepsins, ${ }^{14-16}$ plant xanthohumols ${ }^{17}$ and fungal roselipins ${ }^{18-20}$ (Figure 1). The selectivity of these inhibitors towards the two DGAT isozymes could not be assessed in the rat liver system. In this study, enzyme-based assay systems for DGAT1 and DGAT2 isozymes were established by transforming DGAT-deficient Saccharomyces cerevisiae using complementary DNA (cDNA) of human DGAT isozymes, which allowed us to investigate the selectivity toward the isozymes of natural DGAT inhibitors discovered earlier.

\section{MATERIALS AND METHODS}

Materials

Amidepsines $\mathrm{A}-\mathrm{D}$ and roselipins $1 \mathrm{~A}, 1 \mathrm{~B}, 2 \mathrm{~A}$ and $2 \mathrm{~B}$ were purified from the culture broth of the respective microorganism according to established methods. ${ }^{14,18}$ Xanthohumol was purified from the methanol extracts of Humulus lupulus according to an established method. ${ }^{17}\left[1-{ }^{14} \mathrm{C}\right]$ Palmitoyl-CoA was purchased from GE Healthcare UK Ltd (Amersham Place, Little Chalfont, UK)

\section{General}

Standard molecular biological techniques were applied ${ }^{21}$. For polyethylene glycolmediated transformation of yeast cells, the lithium acetate method was used. ${ }^{22}$

Strains, media and chemicals

S. cerevisiae BY4742-DGA1, a DGAT-deficient mutant of S. cerevisiae BY4742 (MAT $\alpha$ his3 leu2 lys2 ura3), was purchased from Open Biosystems (Huntsville, AL, USA) (www.openbiosystems.com). S. cerevisiae OP3-C (MAT $\alpha$ leu2 ura3) was used for the reference strain of DGAT activity. Escherichia coli strain DH5 $\alpha$ $\left(\mathrm{F}^{-}\right.$Ф80dlacZ $\Delta \mathrm{M} 15 \Delta$ (lacZYA-argF) U169 deoR recAl endA1 hsdR17 ( $\mathrm{rk}^{-}$, $\mathrm{mk}+)$ phoA supE44 $\lambda^{-}$thi-1 gyrA96 relA1) was used in DNA manipulations.

${ }^{1}$ School of Pharmacy, Kitasato University, Tokyo, Japan and ${ }^{2}$ Kitasato Institute for Life Sciences and Graduate School of Infection Control Sciences, Kitasato University, Tokyo, Japan

Correspondence: Dr H Tomoda, School of Pharmacy, Kitasato University, 5-9-1 Shirokane, Minato-ku, Tokyo 108-8641, Japan.

E-mail: tomodah@pharm.kitasato-u.ac.jp

Received 30 July 2008; revised 7 October 2008; accepted 7 October 2008; published online 9 January 2009 
YPD medium (1.0\% bacto-yeast extracts, 2.0\% bacto-peptone, 2.0\% glucose) HeLa cells and a human liver cDNA library (Takara Bio Inc., Shiga, Japan),

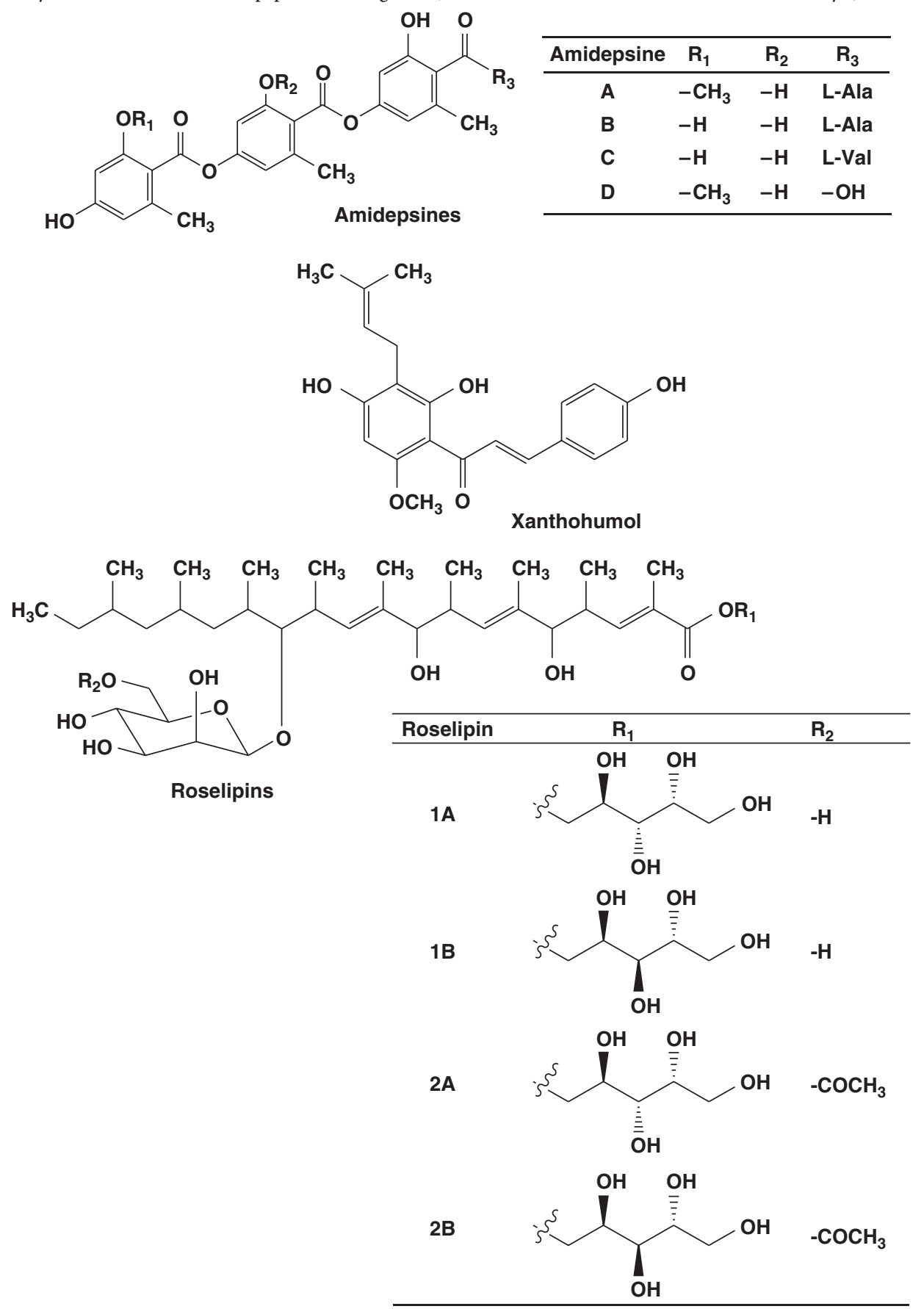

Figure 1 Structures of acyl-CoA:diacylglycerol acyltransferase (DGAT) inhibitors discovered using rat liver microsomes in an enzyme-based assay.

was used to grow non-transformed yeast strains. Selection medium (SM-Glc; yeast nitrogen base w/o amino acids $0.67 \%$, cassamino acid $0.5 \%$, glucose $2.0 \%$ ) was used for the maintenance of yeast strains transformed with pYES, harboring either no insert or DGAT1 or DGAT2 cDNA. Production medium (PM-Gal; yeast nitrogen base w/o amino acid $0.67 \%$, cassamino acid $0.5 \%$, galactose $2.0 \%$ ) was used for production of the recombinant human DGAT isozymes by the transformed yeasts.

\section{Plasmids}

The coding regions of human DGAT1 (accession no. AF059202) and DGAT2 (accession no. AY358532) genes were amplified using cDNA prepared from respectively, by polymerase chain reaction (PCR) (30 cycles, 1 min at $94{ }^{\circ} \mathrm{C}$, $1 \mathrm{~min}$ at $55^{\circ} \mathrm{C}$ and $6 \mathrm{~min}$ at $68^{\circ} \mathrm{C}$ ) using the following oligonucleotide sets: N-terminal primer $5^{\prime}$-GGG GAC AAG TTT GTA CAA AAA AGC AGG CTC CAT GGG CGA CCG CGG CAG C-3' and C-terminal primer 5'-GGG GAC CAC TTT GTA CAA GAA AGC TGG GTA GCT CAG GCC TCT GCC GCT G-3' for DGAT1, and N-terminal primer 5'-GGG GAC AAG TTT GAT CAA AAA AGC AGG CTC CAT GAA GAC CCT CAT AG- ${ }^{\prime}$ and C-terminal primer 5'-GGG GAC CAC TTT GTA CAA GAA AGC TGG GTG CAG CTG GTT CCT CCA GG-3' for DGAT2. The DGAT1 and DGAT2 cDNAs were transferred to pDONR201 (Invitrogen, Carlsbad, CA, USA) between the att1 and att2 sites by site-specific recombination according to the protocols, and the nucleotide 
sequences of the cDNA regions were determined by a PRISM 3100 Genetic Analyzer (Applied Biosystems) using M13 Primer M4 and M13 Primer RV (Takara Bio). The cDNAs were then transferred to pYES-DEST52 (Invitrogen) according to the manufacturer's protocols. The vector carried the URA3 marker for auxotrophic selection and a GAL1 promoter for protein expression.

\section{Expression of the recombinant DGAT1 and DGAT2 in yeasts} and preparation of microsomes

S. cerevisiae BY4742- $\triangle D G A 1$ transformed with pYES, harboring either no insert or DGAT1 or DGAT2 cDNA (designated as mock S. cerevisiae, DGAT1-S. cerevisiae and DGAT2-S. cerevisiae, respectively), was grown for $24 \mathrm{~h}$ in SM-Glc medium. The cells were washed twice with PM-Gal, resuspended in the same medium at $\mathrm{OD}_{590 \mathrm{~nm}}=0.4$ and grown for another $24 \mathrm{~h}$. The cells were washed once with ice-cold PBS(-) and resuspended in DGAT buffer containing $50 \mathrm{mM}$ Tris- $\mathrm{HCl}, 140 \mathrm{mM} \mathrm{KCl}, 0.1 \mathrm{mM}$ EDTA (pH 7.5) and complete Mini (F. Hoffmann-La Roche AG, Basel, Switzerland). Cell lysates obtained by disintegration using a French Press (GLEN MILLS INC., Clifton, NJ, USA) were centrifuged at $15000 \mathrm{~g}$ for $30 \mathrm{~min}$. The supernatant was collected and centrifuged at $105000 \mathrm{~g}$ for $1 \mathrm{~h}$ to precipitate the microsomes. The pellet was resuspended in DGAT buffer and the protein concentration was determined by a BCA protein assay kit (Thermo Fisher Scientific Inc., Rockford, IL, USA). The microsomes were aliquoted and frozen at $-80^{\circ} \mathrm{C}$.

\section{DGAT assay}

Microsomal fractions from mock, DGAT1- and DGAT2-S. cerevisiae were used to measure DGAT activity. Assays were performed in duplicate as described previously. ${ }^{14}$ An assay mixture contained $175 \mathrm{~mm}$ Tris- $\mathrm{HCl}(\mathrm{pH} 8.0$ ), 50-75 $\mu \mathrm{g}$ yeast microsomal protein, $14.5 \mu \mathrm{M}$ bovine serum albumin, $\left[1-{ }^{14} \mathrm{C}\right]$ palmitoyl$\mathrm{CoA}(30 \mu \mathrm{M}, 7.4 \mathrm{kBq}), 8 \mathrm{~mm} \mathrm{MgCl}_{2}, 2.5 \mathrm{~mm}$ diisopropylfluorophosphate and $150 \mu \mathrm{M}$ 1,2-dioleoyl-sn-glycerol; experimental inhibitors were dissolved in $2.5 \mu \mathrm{l}$ of methanol and included in a total assay reaction volume of $0.2 \mathrm{ml}$. The assay was initiated by addition of the microsomal fraction. After incubation at $23^{\circ} \mathrm{C}$ for $15 \mathrm{~min}$, the reaction was stopped by addition of $1.2 \mathrm{ml}$ of chloroform: methanol (1:2) and lipids were extracted according to the method of Folch et al. ${ }^{23}$ TG was separated by TLC on Silica gel 60 plates (Merck Co.) using a petroleum ether:diethylether:acetic acid (80:20:1) solvent system. The TLC plates were exposed to a Fujifilm imaging plate to assess the formation of $\left[{ }^{14} \mathrm{C}\right]$ TG. Imaging signals were visualized and quantified with a BAS 2000 imaging analyzer (FUJIFILM Corporation, Tokyo, Japan).

\section{RESULTS}

\section{Construction of DGAT1- and DGAT2-S. cerevisiae}

To obtain active human DGAT isozymes for the construction of the enzyme-based assay system, DGAT1 and DAGT2 cDNAs were originally isolated from HeLa cells and a cDNA library of human liver using PCR. The deduced amino acid sequence of these clones revealed perfect matches with previously reported sequences (accession nos. AF059202 and AF384161). The coding regions of DGAT1 and DGAT2 were introduced to the yeast expression vector pYES-DES52 by in vitro recombination, and the constructed plasmids were transformed into DGAT-deficient $S$. cerevisiae to establish DGAT1and DGAT2-S. cerevisiae.

\section{DGAT1 and DGAT2 activity}

TG synthesis catalyzed by DGAT in the microsomes prepared from DGAT-deficient $S$. cerevisiae and mock $S$. cerevisiae was measured. As shown in Figure 2, $\left[{ }^{14} \mathrm{C}\right] \mathrm{TG}$ was not detected (lanes 2 and 3), indicating that these mutant yeasts completely lack DGAT activity.

On the other hand, TG synthetic activity was recovered in the microsomes prepared from DGAT1- and DGAT2-S. cerevisiae (lanes 4 and 5). Thus, it is possible to measure human DGAT1 and DGAT2 activities by using these transformed yeasts. The activities of both DGAT isozymes were more potent than that in rat liver microsomes or in the reference yeast strain OP3-C with native yeast DGAT (Figure 2).

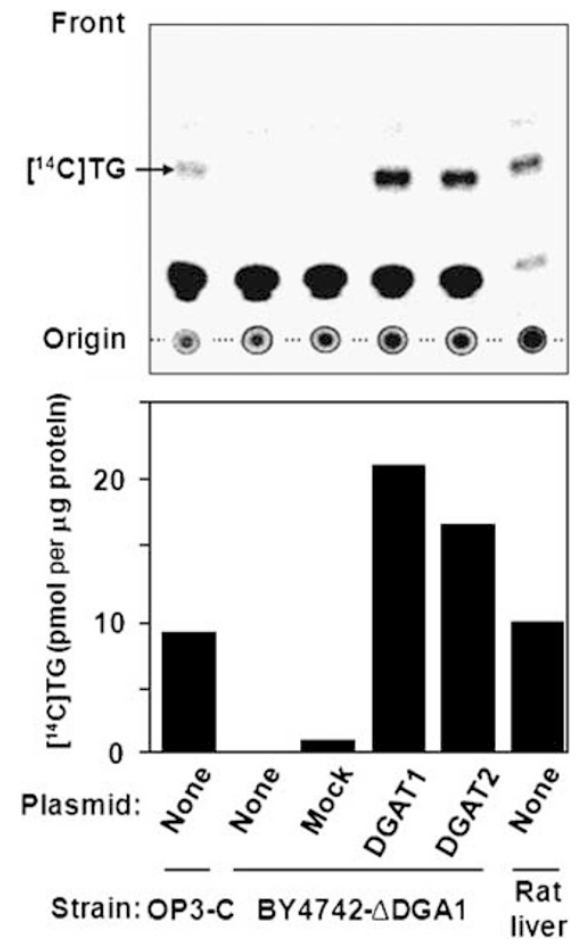

Figure 2 In vitro diacylglycerol esterification by recombinant human DGATs produced in yeast. DGAT activities of membrane fractions from $S$. cerevisiae OP3-C (lane 1), S. cerevisiae BY4742- $\triangle$ DGA1 (lane 2), S. cerevisiae BY4742 transformed with pYES harboring either no insert (lane 3) or DGAT1 (lane 4) or DGAT2 DNA (lane 5) and rat liver (lane 6) were measured. The enzyme reactions and the analysis of the reaction products were performed as described in text.

Table 1 Selectivity of DGAT inhibitors of natural origin toward acyl-CoA:diacylglycerol acyltransferase isozymes

\begin{tabular}{lrrr}
\hline & & \multicolumn{1}{c}{$I C_{50}(\mu \mathrm{M})$} & \\
\cline { 2 - 4 } Compound & DGAT1 & DGAT2 & Rat liver \\
\hline Amidepsine A & 40 & 70 & 10 \\
Amidepsine B & 30 & 60 & 19 \\
Amidepsine C & 200 & 200 & 52 \\
Amidepsine D & 20 & 30 & 18 \\
Xanthohumol & 40 & 40 & 58 \\
Roselipin 1A & 200 & 30 & 17 \\
Roselipin 1B & $>200$ & 40 & 15 \\
Roselipin 2A & $>200$ & 45 & 22 \\
Roselipin 2B & $>200$ & 50 & 18 \\
\hline
\end{tabular}

Abbreviation: DGAT, diacylglycerol acyltransferase.

a Data cited from Matsuda \& Tomoda. ${ }^{8}$

Selectivity of DGAT inhibitors toward the isozymes

The selectivity of the three types of DGAT inhibitors, namely amidepsines, xanthohumol and roselipins (Figure 1), toward the isozymes was tested in the DGAT1 and DGAT2 assays. The $\mathrm{IC}_{50}$ values are summarized in Table $1 . \mathrm{IC}_{50}$ values previously determined in rat liver microsomes are also shown for comparison.

Amidepsines and xanthohumol inhibited both DGAT1 and DGAT2 isozymes with similar potency. The order of potency is similar to that seen with rat liver microsomes. Amidepsine C, which contains a Val 
residue, is less potent than those inhibitors that have an Ala residue (amidepsines A and B) or no amino acid (amidepsine D). On the other hand, roselipins inhibited DGAT2 isozymes with $\mathrm{IC}_{50}$ values of $15-40 \mu \mathrm{M}$, but showed almost no inhibition against DGAT1 even at $200 \mu \mathrm{m}$. Thus, roselipins are found to be selective inhibitors of DGAT2.

\section{DISCUSSION}

In this study, human DGAT1 and DGAT2 genes were transformed into DGAT-deficient $S$. cerevisiae to establish DGAT1- and DGAT2expressing yeasts.

Biosynthesis of TG in S. cerevisiae is mainly catalyzed by the two acyltransferases, DGA1 and LRO1 proteins. DGA1 encodes an acyl CoA:DGAT, which is similar to the MrDGAT2A and MrDGAT2B proteins from Mortierella ramanniana with $\sim 36 \%$ identity, and thus DGA1 protein exhibits acyl-CoA-mediated DG esterification activity. ${ }^{24}$ On the other hand, LRO1 shows a significant sequence similarity to the human lecitin:cholesterol acyltransferase: LRO1 protein mediates esterification of DG using the $s n-2$ acyl group of phospholipids as an acyl donor. ${ }^{25}$

Whether these enzymes would interfere with our assay was tested using assays with microsomes prepared from yeast lacking DGA-1 and from the same strain transformed with vector lacking the cDNA insert. Our results showed that DGAT activity was not detected in DGA1deficient yeast (Figure 2). In contrast, the microsomal fractions prepared from yeasts transformed with cDNA for DGAT1 or DGAT2 showed strong enzyme activity. Using these enzymes we found that amidepsines and xanthohumol inhibit both DGAT1 and DGAT2, but roselipins are DGAT2-selective inhibitors (Table 1). Thus, the assay system is useful for the study of DGAT inhibitors. Furthermore, it is plausible that rat liver microsomes contain DGAT1 and DGAT2 isozymes.

\section{ACKNOWLEDGEMENTS}

This work was supported by a grant-in-aid for Scientific Research on Priority Areas 16073215 (to HT) and Scientific Research (B) 18390008 (to HT) from the Ministry of Education, Culture, Sports, Science and Technology, Japan.

1 Coleman, R. A., Lewin, T. M. \& Muoio, D. M. Physiological and nutritional regulation of enzymes of triacylglycerol synthesis. Annu. Rev. Nutr. 20, 77-103 (2000).
2 Bell, R. M. \& Coleman, R. A. Enzymes of glycerolipid synthesis in eukaryotes. Ann. Rev. Biochem. 49, 459-487 (1980).

3 Kahn, C. R. Triglycerides and toggling the tummy. Nat. Genet. 25, 6-7 (2000).

4 Cases, S. et al. Identification of a gene encoding an acyl CoA:diacylglycerol acyltransferase, a key enzyme in triacylglycerol synthesis. Proc. Natl Acad. Sci. USA 95, 13018-13023 (1998).

5 Oelkers, P., Behari, A., Cromley, D., Billheimer, J. T. \& Sturley, S. L. Characterization of two human genes encoding acyl coenzyme A:cholesterol acyltransferase-related enzymes. J. Biol. Chem. 273, 26765-26771 (1998).

6 Cases, S. et al. Cloning of DGAT2, a second mammalian diacylglycerol acyltransferase, and related family members. J. Biol. Chem. 276, 38870-38876 (2001).

7 Tomoda, H. \& Ōmura, S. Potential therapeutics for obesity and atherosclerosis: inhibitors of neutral lipid metabolism from microorganisms. Pharmacol. Ther. 115, 375-389 (2007)

8 Matsuda, D. \& Tomoda, H. DGAT inhibitors for obesity. Curr. Opin. Invest. Drugs 8, 836-841 (2007)

9 Smith, S. J. et al. Obesity resistance and multiple mechanisms of triglyceride synthesis in mice lacking DGAT. Nat. Genet. 25, 87-90 (2000).

10 Chen, H. C. et al. Increased insulin and leptin sensitivity in mice lacking acyl CoA:diacylglycerol acyltransferase 1. J. Clin. Invest. 109, 1049-1055 (2002).

11 Stone, S. J. et al. Lipopenia and skin barrier abnormalities in DGAT2-deficient mice. J. Biol. Chem. 279, 11767-11776 (2004).

12 Yamazaki, T. et al. Increased very low density lipoprotein secretion and gonadal fat mass in mice overexpressing liver DGAT1. J. Biol. Chem. 280, 21506-21514 (2005).

13 Turkish, A. \& Sturley, S. L. Regulation of triglyceride metabolism. I. Eukaryotic neutral lipid synthesis: 'Many ways to skin ACAT or a DGAT'. Am. J. Physiol. Gastrointest. Liver Physiol. 292, G953-G957 (2007).

14 Tomoda, H. et al. Amidepsines, inhibitors of diacylglycerol acyltransferase produced by Humicola sp. FO-2942. I. Production, isolation and biological properties. J. Antibiot. 48, 937-941 (1995)

15 Tomoda, H., Tabata, N., Ito, M. \& Ōmura, S. Amidepsines, inhibitors of diacylglycerol acyltransferase produced by Humicola sp. FO-2942. II. Structure elucidation of amidepsines A, B and C. J. Antibiot. 48, 942-947 (1995).

16 Tomoda, H. et al. Amidepsine E, an inhibitor of diacylglycerol acyltransferase produced by Humicola sp. FO-5969. J. Antibiot. 49, 929-931 (1996).

17 Tabata, N., Ito, M., Tomoda, H. \& Ōmura, S. Xanthohumols, diacylglycerol acyltransferase inhibitors, from Humulus lupulus. Phytochemistry 46, 683-687 (1997).

18 Tomoda, H. et al. Roselipins, inhibitors of diacylglycerol acyltransferase, produced by Gliocladium roseum KF-1040. J. Antibiot. 52, 689-694 (1999).

19 Tabata, N. et al. Structure elucidation of roselipins, inhibitors of diacylglycerol acyltransferase produced by Gliocladium roseum KF-1040. J. Antibiot. 52, 815-826 (1999).

20 Tomoda, H., Tabata, N., Ohyama, Y. \& Ōmura, S. Core structure in roselipins essential for eliciting inhibitory activity against diacylglycerol acyltransferase. J. Antibiot. 56, 24-29 (2003).

21 Sambrook, J., Fritsch, E. F. \& Maniatis, T. Molecular Cloning: A Laboratory Manual 2nd edn (Cold Spring Harbor Laboratory Press, Cold Spring Harbor, NY, 1989).

22 Ito, H., Fukuda, Y., Murata, K. \& Kimura, A. Transformation of intact yeast cells treated with alkali cations. J. Bacteriol. 153, 163-168 (1983).

23 Folch, J., Lees, M. \& Sloane Stanley1, G. H. A simple method for the isolation and purification of total lipids from animal tissues. J. Biol. Chem. 226, 497-509 (1957).

24 Oelkers, P., Cromley, D., Padamsee, M., Billheimer, J. T. \& Sturley, S. L. The DGA1 gene determines a second triglyceride synthetic pathway in yeast. J. Biol. Chem. 277, 8877-8881 (2002).

25 Sandager, L. et al. Storage lipid synthesis is non-essential in yeast. J. Biol. Chem. 277, 6478-6482 (2002). 